\title{
Editorial
}

\section{Contribution of Pore-Scale Approach to Macroscale Geofluids Modelling in Porous Media}

\author{
Emanuele Romano ${ }^{(D)}{ }^{1}$ Joaquín Jiménez-Martínez, ${ }^{2}$ Andrea Parmigiani, ${ }^{3}$ \\ Xiang-Zhao Kong $\mathbb{D}^{4}{ }^{4}$ and Ilenia Battiato ${ }^{5}$ \\ ${ }^{1}$ Water Research Institute, National Research Council of Italy, Area della Ricerca-Roma 1, via Salaria km 29, \\ 300-00015 Montelibretti, Italy \\ ${ }^{2}$ Subsurface Environmental Processes Group, Eawag and ETH Zurich, Zurich, Switzerland \\ ${ }^{3}$ Institute of Geochemistry and Petrology, ETH Zurich, Clausiusstrasse 25, 8092 Zurich, Switzerland \\ ${ }^{4}$ Geothermal Energy and Geofluids Group, Institute of Geophysics, ETH Zurich, 8092 Zurich, Switzerland \\ ${ }^{5}$ Energy Resources Engineering, Stanford University, 450 Serra Mall, Stanford, CA 94305, USA \\ Correspondence should be addressed to Emanuele Romano; romano@irsa.cnr.it
}

Received 28 April 2019; Accepted 28 April 2019; Published 22 May 2019

Copyright (c) 2019 Emanuele Romano et al. This is an open access article distributed under the Creative Commons Attribution License, which permits unrestricted use, distribution, and reproduction in any medium, provided the original work is properly cited.

\section{Motivations and Background}

Understanding the fundamental mechanisms of fluid flows and reactive transport in natural systems is a major challenge for several fields of Earth sciences (e.g., hydrology, soil science, and volcanology) and geo/environmental engineering $\left(\mathrm{CO}_{2}\right.$ sequestration, NAPLS contamination, geothermal energy, and oil and gas reservoir exploitation).

The hierarchical structures of natural system (e.g., heterogeneity of geological formations) as well as the different behavior of single and multiphase fluids at the pore-scale coupled with the nonlinearity of underlying reactive processes necessitates investigating these aspects at the scale at which they physically occur, the scale of pore and fractures.

Recent improvements in pore-scale computational modelling, together with the development of noninvasive microscopic imaging technology and the latest microfluidic technics are allowing the vast field of porous and fractured media research to benefit of major advances due to (1) an improved understanding and description of porescale mechanisms and (2) the ability of thinking in terms of coupled processes.

The contributions collected in this special issue, although far from constituting a comprehensive picture of the "porescale world," however offer a good example of the potentialities of such an approach to investigate a wide range of processes usually observed at macroscale, but whose underlying physical and chemical processes take place at microscale.

\section{Contents of the Special Issue}

While a number of different porous media and chemicalphysical processes have been investigated in the papers collected in this special issue, we think that there is a common point shared by all the contributions: the attempt to assess sound criteria to upscale information. Even if not always explicitly stated, all studies try to answer the following question: which kind of information we should collect at microscale to improve our understanding and therefore forecasting of processes observed at macroscale?

In the following, a short presentation of each contribution is given.

(1) "Fluid Interfaces during Viscous-Dominated Primary Drainage in 2D Micromodels Using Pore-Scale SPH Simulations" by R. Sivanesapillai and H. Steeb. Authors adopt a pore-scale hydrodynamic direct numerical approach to investigate viscous-dominated two-phase flow. In particular, simulations of primary drainage in partially wettable $2 \mathrm{D}$ porous media of particulate 
microstructure at large capillary numbers have been performed. Numerical results indicate that the characteristic length of the capillary dispersion zone increases with the heterogeneity of the microstructure.

(2) "Upscaling Strategies of Porosity-Permeability Correlations in Reacting Environments from Pore-Scale Simulations" by N. I. Prasianakis et al. The paper focuses on one of the still open challenges in geochemically reacting environments, i.e., the modeling and upscaling of porosity and permeability changes due to mineral dissolution and precipitation processes. Permeability-porosity correlations, extracted after fitting known type of relationships, are highly nonlinear and strongly dependent on the magnitude of Péclet and Damköhler numbers. The authors finally discuss the challenges of upscaling while curbing computational costs.

(3) "Quantify the Pore Water Velocity Distribution by a Celerity Function" by W. Shao et al. Fluid flow velocities within porous media show a widely heterogeneous distribution with the coexistence of high velocities, also call preferential paths, and low velocity regions or stagnations zones. W. Shao et al. propose to quantify the pore water velocity distribution from a celerity function. They showed that the proposed function can assist in investigating subsurface flow and tracer transport, both in fully and partially saturated conditions, and the kinematic ratio could be used to predict the first arrival time of a conservative tracer.

(4) "Effect of Pore-Scale Mineral Spatial Heterogeneity on Chemically Induced Alterations of Fractured Rock: A Lattice Boltzmann Study" by H. Fazeli et al. Authors investigate the pore-scale porosity-permeability relationship during mineral dissolution reactions in a multimineral fracture with a focus on a well-mixed and a banded structure, using lattice-Boltzmann methods. Authors identify a degraded zone in the well-mixed structures due to the dissolution of fastdissolving minerals, and a comb-tooth zone in the banded structures. They finally conclude that besides the contribution of geometry structures, the porositypermeability relationship greatly relies on the values of Péclet and Damköhler numbers.

(5) "The Visual and Quantitative Study of the Microoccurrence of Irreducible Water at the Pore and Throat System in a Low-Permeability Sandstone Reservoir by Using Microcomputerized Tomography" by X. Gu et al. With 3D tomographic imaging on a low permeable sandstone after a displacement of brine with oil, authors observed that the irreducible water (wetting phase) tends to accumulate in the throat system as water films, leading to a significant cutoff of drainage channels of oil. While the remaining irreducible water spreads in the corners of the pore system, these water blobs are primarily linked through a water film. These findings advance the understandings on irreducible water microbehaviors.

(6) "A Fluid-Solid Coupling Mathematical Model of Methane Driven by Water in Porous Coal" by B. Huang and $W$. Lu. The mathematical coupling of multiphase flow and adsorption-desorption processes within porous media is of extraordinary complexity. The fluid-solid model proposed by B. Huang and $\mathrm{W}$. Lu has demonstrated its potential in reproducing experimental result of water-driven methane in porous coal.

(7) "Study on Reasonable Energy Supplement Time of Tight Sandstone Oil Reservoirs with Rock Compressibility Stress Sensitivity" by T. Xiaofeng et al. T. Xiaofeng et al. Proposes a new method to estimate the energy supplement time (i.e., the time when it is convenient to supply energy for extraction because the natural energy is going to decrease) in an oil reservoir. Such an approach, validated on experimental data, takes into account at the pore scale of both threshold pressure gradient and rock compressibility stress sensitivity, demonstrating that the latter cannot be neglected.

(8) "Permeability Estimation Based on the Geometry of Pore Space via Random Walk on Grids" by T. Nan et al. The characterization of hydraulic properties is always challenging because of its strong dependence on geometry and topology. In this special issue, $\mathrm{T}$. Nan et al. introduce a method to estimate permeability based on the geometry of pore space via random walks on grids. This method overcomes difficulties found by widely used approaches such as "walk on spheres" or "walk on cubes." In comparison with Green's functions for simple geometries and computational fluid-flow dynamics for complex geometries, the proposed method is invaluable in the estimation of permeability from digitalized porous media.

(9) "Impact of Synthetic Porous Medium Geometric Properties on Solute Transport Using Direct 3D Pore-Scale Simulations" by P. R. Di Palma et al. The study proposes a systematic analysis of the relationships between geometric features of the porous media, as defined in the context of Minkowski functionals, and transport processes through direct simulations of fluid flow and advection-diffusion of a nonreactive solute in synthetically generated 3D media. The study finds that the advective transport is primarily affected by the first and second Minkowski functional, while effective diffusion scales as the inverse of the tortuosity squared.

\section{Next Challenges}

We believe that in the next future, advances in understanding physical and chemical processes occurring in porous media will take advantage from the superposition of approaches 
able to collect information at different scales, from nano to macro. Such a multiscale approach will be favoured by the increasingly performing imaging techniques, as well as by the increasing computational power allowing for a number of "numerical experiments" much larger than in the past.

Therefore, to conclude this short Editorial, we wish to suggest some "research challenges" that may be addressed in the next future.

(1) Scale Translation in Multiphase Reactive Systems. Notwithstanding the progress made in forward predictions of flow and reactive transport in porous media over the past decades, the achievement of a seamless predictive understanding of the system response at the macroscale (be it Darcy or field scale) from pore-scale processes appears still elusive, especially when the scale disparity between adjacent models increases (e.g., from pore scale to Darcy scale versus from pore scale to field scale) [1]. Such challenges are further amplified in reactive multiphase systems, where the complex distribution of phases, as well as the inherent instability of the interfaces separating them, undermine the very foundation of some of the upscaling methods classically employed to build effective medium models $[2,3]$. Despite the ubiquity of multiphase flows in geologic media, current modelling efforts are still based on postulated multiphase Darcy's equations, whose limitations have been ostensibly highlighted by numerous studies, and alternative physics-based formulations $[4,5]$ are still slow to penetrate common wisdom. This can greatly affect one's ability to properly model reaction rates, distribution of contaminants and resources (gas and oil), nutrient cycling, etc., in multiphase systems. Moving away from parametrization of effective models toward estimation of effective properties from pore-scale models in a bottom-up framework requires the development of holistic approaches which combine rigorous upscaling, numerical simulations, experiments, and, last but not least, data analytics tools, while ensuring that predictive errors remain bounded by formal upscaling errors. The originally parallel paths between big data analytics and physics-based models must now intersect to generate the longed leap-frog improvement in predictive understanding of systems dominated by a tyranny of scales like porous media.

(2) Validation with $4 D$ Experiments. Challenges should also be focused on validation of numerical predictions [6] on pore-scale processes using wellcontrolled laboratory experiments $[7,8]$. It has been extremely difficult to visualize 3D time-series (i.e., 4D) data during laboratory experiments that investigate reactive transport through a porous and/or fractured medium at sufficient spatial and temporal resolutions, because the processes of interest occur (deep) inside the medium of interest. Thanks to fast evolution of nondestructive and noninvasive instrumentation and detectors as well as the development of ultrafast imaging procedures, a micrometer to nanometer resolution is becoming affordable even during tracking of very rapid dynamic processes in porous media. The real-time tracking of pore-scale multiphase reactive transport processes enables confirmation/validation/reexamination of known mechanisms and reveal/discover unknown mechanisms, offering parametrization of the existing models and establishment of novel approaches to include the newly discovered mechanisms.

(3) Fluid-Biofilm Interactions in Porous Media. Processes of biofilm formation play a fundamental role in several natural phenomena, as well as in technological applications, ranging from bioreactors, to permeable reactive barriers, to medical systems. Biofilm growth is a stepwise complex process from attachment and adaptation to detachment of cells and biofilm fragments whose evolution is affected by and at the same time affects fluid dynamics features at the pore scale [9]. Such a strong interaction between biological processes and microfluidics is very far from being understood. Insights for a deeper comprehension of the mechanisms (mechanical and biological) involved in the biofilm growth can arise from a multidisciplinary approach involving (1) laboratory microfluidic experiments under flow controlled conditions [10], (2) noninvasive imaging methods for assessing the biomass evolution [11], and (3) 3D numerical models [12] coupling biofilm growth and pore-scale fluid dynamics models [13].

\section{Conflicts of Interest}

The guest editors declare that they have no conflicts of interest or private agreements with companies.

\section{Acknowledgments}

We sincerely thank Paolo Roberto Di Palma for supporting the editorial work in this special issue.

Emanuele Romano

Joaquín Jiménez-Martínez

Andrea Parmigiani

Xiang-Zhao Kong

Ilenia Battiato

\section{References}

[1] P. de Anna, J. Jiménez-Martínez, H. Tabuteau et al., "Mixing and reaction kinetics in porous media: an experimental pore scale quantification," Environmental Science \& Technology, vol. 48, no. 1, pp. 508-516, 2014.

[2] J. Jiménez-Martínez, P. de Anna, H. Tabuteau, R. Turuban, T. Le Borgne, and Y. Méheust, "Pore-scale mechanisms for the enhancement of mixing in unsaturated porous media and implications for chemical reactions," Geophysical Research Letters, vol. 42, no. 13, pp. 5316-5324, 2015. 
[3] J. Jiménez-Martínez, T. Le Borgne, H. Tabuteau, and Y. Méheust, "Impact of saturation on dispersion and mixing in porous media: photo-bleaching pulse injection experiments and shear-enhanced mixing model," Water Resources Research, vol. 53, no. 2, pp. 1457-1472, 2017.

[4] D. Picchi and I. Battiato, "The impact of pore-scale flow regimes on upscaling of immiscible two-phase flow in porous media," Water Resources Research, vol. 54, no. 9, pp. 66836707, 2018.

[5] D. Picchi and I. Battiato, "Relative permeability scaling from pore-scale flow regimes," Water Resources Research, vol. 55, 2019.

[6] A. Parmigiani, P. Di Palma, S. Leclaire, F. Habib, and X.-Z. Kong, "Characterization of transport-enhanced phase separation in porous media using a lattice-Boltzmann method," Geofluids, vol. 2019, 2019.

[7] M. Ahkami, T. Roesgen, M. O. Saar, and X.-Z. Kong, "Highresolution Temporo-ensemble PIV to resolve pore-scale flow in 3D-printed fractured porous media," Transport in Porous Media, pp. 1-17, 2018.

[8] M. Nooraiepour, H. Fazeli, R. Miri, and H. Hellevang, "Effect of $\mathrm{CO}_{2}$ phase states and flow rate on salt precipitation in shale caprocks-a microfluidic study," Environmental Science \& Technology, vol. 52, no. 10, pp. 6050-6060, 2018.

[9] C. D. Persat, M. K. Nadell, F. Kim et al., "The mechanical world of bacteria," Cell, vol. 161, no. 5, pp. 988-997, 2015.

[10] Y. Yawata, J. Nguyen, R. Stocker, and R. Rusconi, "Microfluidic studies of biofilm formation in dynamic environments," Journal of Bacteriology, vol. 198, no. 19, pp. 2589-2595, 2016.

[11] M. Carrel, M. A. Beltran, V. L. Morales et al., "Biofilm imaging in porous media by laboratory X-ray tomography: combining a non-destructive contrast agent with propagation-based phase-contrast imaging tools," PLoS One, vol. 12, no. 7, article e0180374, 2017.

[12] P. R. Di Palma, N. Guyennon, F. Hesse, and E. Romano, "Porous media flux sensitivity to pore-scale geostatistics: a bottom-up approach," Advances in Water Resources, vol. 102, pp. 99-110, 2017.

[13] M. Benioug, F. Golfier, C. Oltéan, M. A. Buès, T. Bahar, and J. Cuny, "An immersed boundary-lattice Boltzmann model for biofilm growth in porous media," Advances in Water Resources, vol. 107, pp. 65-82, 2017. 

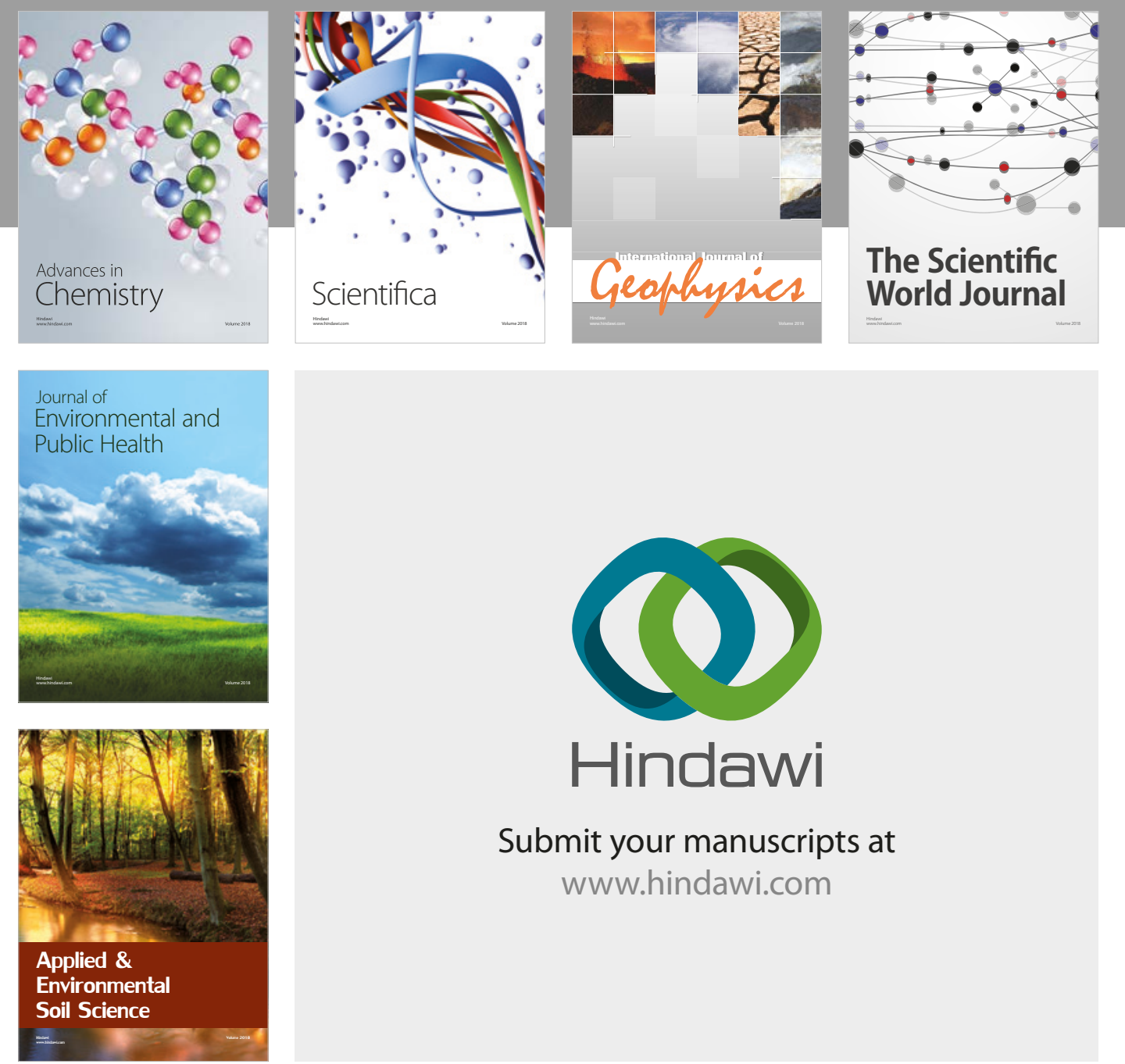

The Scientific

\section{World Journal}
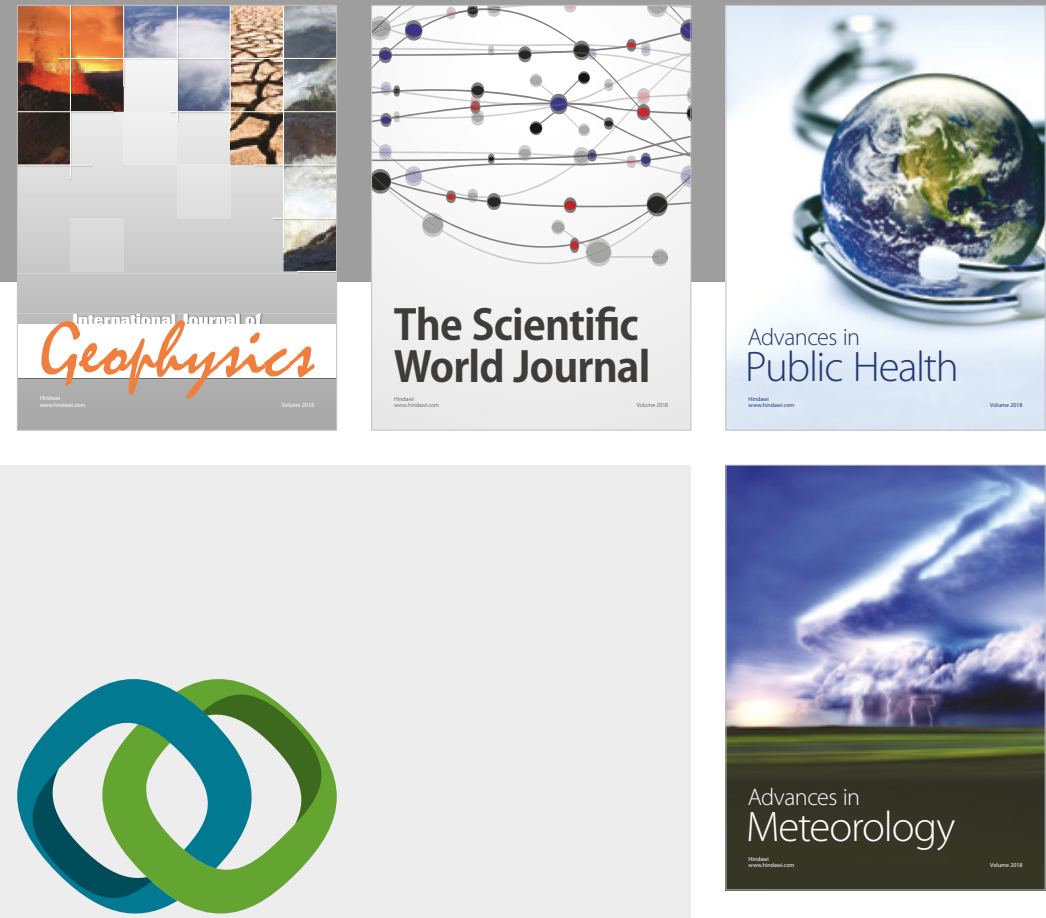

Advan

Public Health

\section{Hindawi}

Submit your manuscripts at

www.hindawi.com
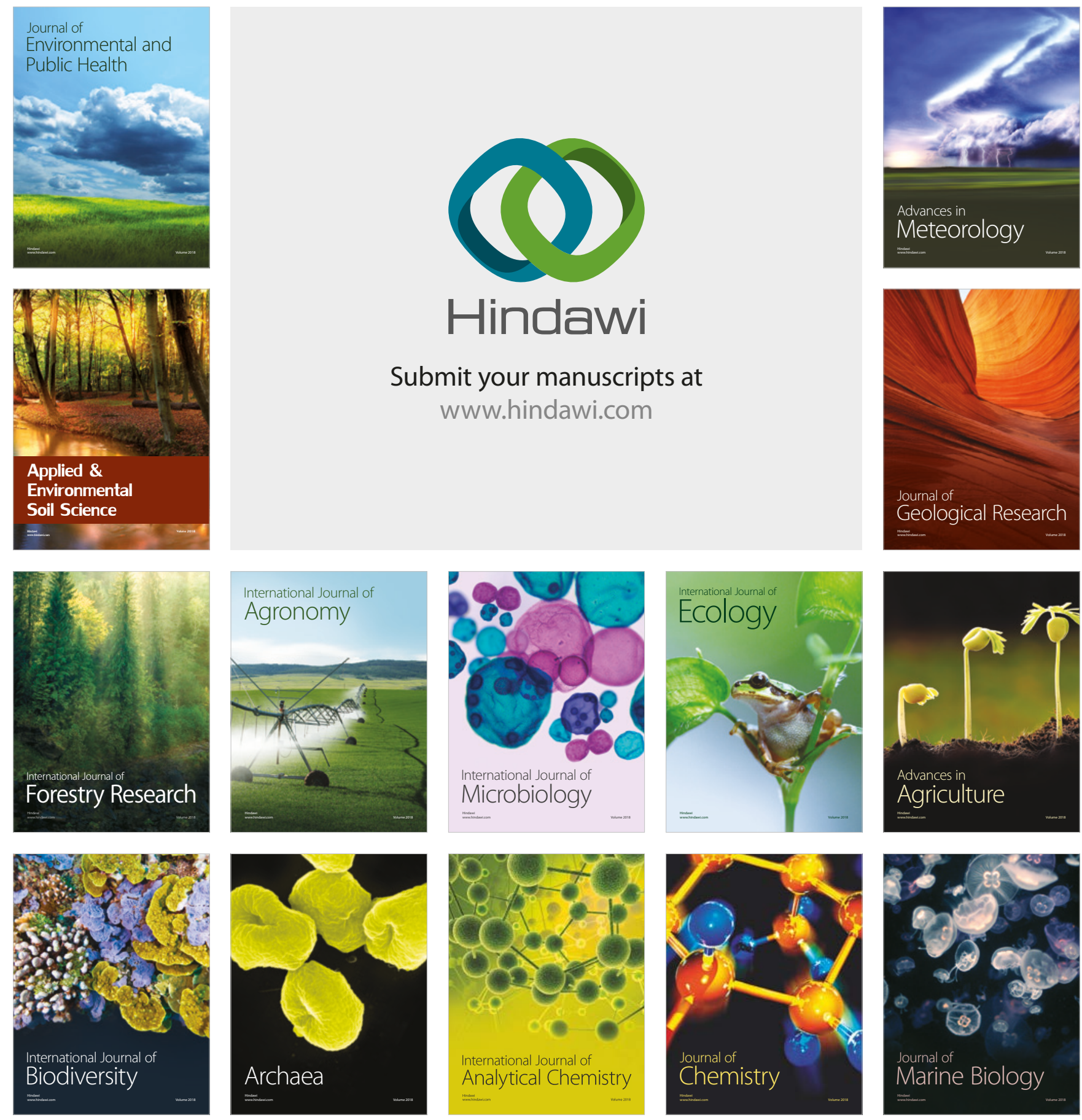